\title{
Apostol-Euler polynomials arising from umbral calculus
}

Taekyun Kim" ${ }^{*}$, Toufik Mansour², Seog-Hoon Rim³ and Sang-Hun Lee ${ }^{4}$

"Correspondence: tkkim@kw.ac.kr 1 Department of Mathematics, Kwangwoon University, Seoul, S. Korea

Full list of author information is available at the end of the article

\begin{abstract}
In this paper, by using the orthogonality type as defined in the umbral calculus, we derive an explicit formula for several well-known polynomials as a linear combination of the Apostol-Euler polynomials.
\end{abstract}

MSC: 05A40

Keywords: Bernoulli polynomial; Bessel polynomial; Euler polynomial; Frobenius-Euler polynomial; umbral calculus

\section{Introduction}

Let $\Pi_{n}$ be the set of all polynomials in a single variable $x$ over the complex field $\mathbb{C}$ of degree at most $n$. Clearly, $\Pi_{n}$ is a $(n+1)$-dimensional vector space over $\mathbb{C}$. Define

$$
\mathcal{H}=\left\{f(t)=\sum_{k \geq 0} a_{k} \frac{t^{k}}{k !} \mid a_{k} \in \mathbb{C}\right\}
$$

to be the algebra of formal power series in a single variable $t$. As is known, $\langle L \mid p(x)\rangle$ denotes the action of a linear functional $L \in \mathcal{H}$ on a polynomial $p(x)$, and we remind that the vector space on $\Pi_{n}$ is defined by

$$
\left\langle c L+c^{\prime} L^{\prime} \mid p(x)\right\rangle=c\langle L \mid p(x)\rangle+c^{\prime}\left\langle L^{\prime} \mid p(x)\right\rangle
$$

for any $c, c^{\prime} \in \mathbb{C}$ and $L, L^{\prime} \in \mathcal{H}$ (see [1-4]). The formal power series in variable $t$ define a linear functional on $\Pi_{n}$ by setting

$$
\left.\left\langle f(t) \mid x^{n}\right\rangle=a_{n} \quad \text { for all } n \geq 0 \text { (see }[1-4]\right) \text {. }
$$

By (1.1) and (1.2), we have

$$
\left\langle t^{k} \mid x^{n}\right\rangle=n ! \delta_{n, k} \quad \text { for all } n, k \geq 0(\text { see }[1-4]),
$$

where $\delta_{n, k}$ is the Kronecker symbol. Let $f_{L}(t)=\sum_{k \geq 0}\left\langle L \mid x^{k}\right\rangle \frac{t^{k}}{k !}$ with $L \in \mathcal{H}$. From (1.3), we have $\left\langle f_{L}(t) \mid x^{n}\right\rangle=\left\langle L \mid x^{n}\right\rangle$. So, the map $L \mapsto f_{L}(t)$ is a vector space isomorphic from $\Pi_{n}$ onto $\mathcal{H}$. Henceforth, $\mathcal{H}$ is thought of as a set of both formal power series and linear functionals. We call $\mathcal{H}$ umbral algebra. The umbral calculus is the study of umbral algebra.

○2013 Kim et al.; licensee Springer. This is an Open Access article distributed under the terms of the Creative Commons Attribution License (http://creativecommons.org/licenses/by/2.0), which permits unrestricted use, distribution, and reproduction in any medium, provided the original work is properly cited. 
Let $f(t) \in \mathcal{H}$. The smallest integer $k$ for which the coefficient of $t^{k}$ does not vanish is called the order of $f(t)$ and is denoted by $O(f(t))$ (see [1-4]). If $O(f(t))=1, O(f(t))=0$, then $f(t)$ is called a delta, an invertible series, respectively. For given two power series $f(t), g(t) \in \mathcal{H}$ such that $O(f(t))=1$ and $O(g(t))=0$, there exists a unique sequence $S_{n}(x)$ of polynomials with $\left\langle g(t)(f(t))^{k} \mid S_{n}(x)\right\rangle=n ! \delta_{n, k}$ (this condition sometimes is called orthogonality type) for all $n, k \geq 0$. The sequence $S_{n}(x)$ is called the Sheffer sequence for $(g(t), f(t))$ which is denoted by $S_{n}(x) \sim(g(t), f(t))$ (see [1-4]).

For $f(t) \in \mathcal{H}$ and $p(x) \in \Pi$, we have

$$
\left\langle e^{y t} \mid p(x)\right\rangle=p(y), \quad\langle f(t) g(t) \mid p(x)\rangle=\langle f(t) \mid g(t) p(x)\rangle
$$

and

$$
f(t)=\sum_{k \geq 0}\left\langle f(t) \mid x^{k}\right\rangle \frac{t^{k}}{k !}, \quad p(x)=\sum_{k \geq 0}\left\langle t^{k} \mid p(x)\right\rangle \frac{x^{k}}{k !}
$$

(see [1-4]). From (1.5), we derive

$$
\left\langle t^{k} \mid p(x)\right\rangle=p^{(k)}(0), \quad\left\langle 1 \mid p^{(k)}(x)\right\rangle=p^{(k)}(0),
$$

where $p^{(k)}(0)$ denotes the $k$ th derivative of $p(x)$ with respect to $x$ at $x=0$. Let $S_{n}(x) \sim$ $(g(t), f(t))$. Then we have

$$
\frac{1}{g(\bar{f}(t))} e^{y \bar{f}(t)}=\sum_{k \geq 0} S_{k}(y) \frac{t^{k}}{k !}
$$

for all $y \in \mathbb{C}$, where $\bar{f}(t)$ is the compositional inverse of $f(t)$ (see [1-6]).

For $\lambda \in \mathbb{C}$ with $\lambda \neq-1$, the Apostol-Euler polynomials (see [7-10]) are defined by the generating function to be

$$
\frac{2}{\lambda e^{t}+1} e^{x t}=\sum_{k \geq 0} E_{k}(x \mid \lambda) \frac{t^{k}}{k !}
$$

In particular, $x=0, E_{n}(0 \mid \lambda)=E_{n}(\lambda)$ is called the $n$th Apostol-Euler number. From (1.8), we can derive

$$
E_{n}(x \mid \lambda)=\sum_{k=0}^{n}\left(\begin{array}{l}
n \\
k
\end{array}\right) E_{n-k}(\lambda) x^{k}
$$

By (1.9), we have $\frac{d}{d x} E_{n}(x \mid \lambda)=n E_{n-1}(x \mid \lambda)$. Also, from (1.8) we have

$$
\frac{2}{\lambda e^{t}+1}=e^{E(\lambda) t}=\sum_{n \geq 0} E_{n}(\lambda) \frac{t^{n}}{n !}
$$

with the usual convention about replacing $E^{n}(\lambda)$ by $E_{n}(\lambda)$. By (1.10), we get

$$
2=e^{E(\lambda) t}\left(\lambda e^{t}+1\right)=\lambda e^{(E(\lambda)+1) t}+e^{E(\lambda) t}=\sum_{n \geq 0}\left(\lambda(E(\lambda)+1)^{n}+E_{n}(\lambda)\right) \frac{t^{n}}{n !} .
$$


Thus, by comparing the coefficients of the both sides, we have

$$
\lambda(E(\lambda)+1)^{n}+E_{n}(\lambda)=2 \delta_{n, 0} .
$$

As is well known, the Bernoulli polynomial (see [11-14]) is also defined by the generating function to be

$$
\frac{t}{e^{t}-1} e^{x t}=\sum_{k \geq 0} B_{k}(x) \frac{t^{k}}{k !}
$$

In the special case, $x=0, B_{n}(0)=B_{n}$ is called the $n$th Bernoulli number. By (1.12), we get

$$
B_{n}(x)=\sum_{k=0}^{n}\left(\begin{array}{l}
n \\
k
\end{array}\right) B_{n-k} x^{k}
$$

From (1.12), we note that

$$
\frac{t}{e^{t}-1}=e^{B t}=\sum_{n \geq 0} B_{n} \frac{t^{n}}{n !}
$$

with the usual convention about replacing $B^{n}$ by $B_{n}$. By (1.13) and (1.14), we get

$$
t=e^{B t}\left(e^{t}-1\right)=e^{(B+1) t}-e^{B t}=\sum_{n \geq 0}\left((B+1)^{n}-B_{n}\right) \frac{t^{n}}{n !},
$$

which implies

$$
B_{n}(1)-B_{n}=(B+1)^{n}-B_{n}=\delta_{n, 1}, \quad B_{0}=1 .
$$

Euler polynomials (see $[4,11,13,15])$ are defined by

$$
\frac{2}{e^{t}+1} e^{x t}=\sum_{k \geq 0} E_{k}(x) \frac{t^{k}}{k !}
$$

In the special case, $x=0, E_{n}(0)=E_{n}$ is called the $n$th Euler number. By (1.16), we get

$$
\frac{2}{e^{t}+1}=e^{E t}=\sum_{n \geq 0} E_{n} \frac{t^{n}}{n !}
$$

with the usual convention about replacing $E^{n}$ by $E_{n}$. By (1.16) and (1.17), we get

$$
2=e^{E t}\left(e^{t}+1\right)=e^{(E+1) t}+e^{E t}=\sum_{n \geq 0}\left((E+1)^{n}+E_{n}\right) \frac{t^{n}}{n !},
$$

which implies

$$
E_{n}(1)+E_{n}=(E+1)^{n}+E_{n}=2 \delta_{n, 0} .
$$


For $\lambda \in \mathbb{C}$ with $\lambda \neq-1$, the Frobenius-Euler (see [11,16-19]) polynomials are defined by

$$
\frac{1+\lambda}{e^{t}+\lambda} e^{x t}=\sum_{k \geq 0} F_{k}(x \mid-\lambda) \frac{t^{k}}{k !}
$$

In the special case, $x=0, F_{n}(0 \mid-\lambda)=F_{n}(-\lambda)$ is called the $n$th Frobenius-Euler number (see [17]). By (1.19), we get

$$
\frac{1+\lambda}{e^{t}+\lambda}=e^{F t}=\sum_{n \geq 0} F_{n}(-\lambda) \frac{t^{n}}{n !}
$$

with the usual convention about replacing $F^{n}(-\lambda)$ by $F_{n}(-\lambda)$ (see [17]). By (1.19) and (1.20), we get

$$
1+\lambda=e^{F(-\lambda) t}\left(e^{t}+\lambda\right)=e^{(F(-\lambda)+1) t}+\lambda e^{F(-\lambda) t}=\sum_{n \geq 0}\left((F(-\lambda)+1)^{n}+\lambda F_{n}(-\lambda)\right) \frac{t^{n}}{n !},
$$

which implies

$$
\lambda F_{n}(-\lambda)+F_{n}(1 \mid-\lambda)=\lambda F_{n}(-\lambda)+(F(-\lambda)+1)^{n}=(1+\lambda) \delta_{n, 0} .
$$

In the next section, we present our main theorem and its applications. More precisely, by using the orthogonality type, we write any polynomial in $\Pi_{n}$ as a linear combination of the Apostol-Euler polynomials. Several applications related to Bernoulli, Euler and FrobeniusEuler polynomials are derived.

\section{Main results and applications}

Note that the set of the polynomials $E_{0}(x \mid \lambda), E_{1}(x \mid \lambda), \ldots, E_{n}(x \mid \lambda)$ is a good basis for $\Pi_{n}$. Thus, for $p(x) \in \Pi_{n}$, there exist constants $c_{0}, c_{1}, \ldots, c_{n}$ such that $p(x)=\sum_{k=0}^{n} c_{k} E_{k}(x \mid \lambda)$. Since $E_{n}(x \mid \lambda) \sim\left(\left(1+\lambda e^{t}\right) / 2, t\right)$ (see (1.7) and (1.8)), we have

$$
\left\langle\frac{1+\lambda e^{t}}{2} t^{k} \mid E_{n}(x \mid \lambda)\right\rangle=n ! \delta_{n, k}
$$

which gives

$$
\left\langle\frac{1+\lambda e^{t}}{2} t^{k} \mid p(x)\right\rangle=\sum_{\ell=0}^{n} c_{\ell}\left\langle\frac{1+\lambda e^{t}}{2} t^{k} \mid E_{\ell}(x \mid \lambda)\right\rangle=\sum_{\ell=0}^{n} c_{\ell} \ell ! \delta_{\ell, k}=k ! c_{k} .
$$

Hence, we can state the following result.

Theorem 2.1 For all $p(x) \in \Pi_{n}$, there exist constants $c_{0}, c_{1}, \ldots, c_{n}$ such that $p(x)=$ $\sum_{k=0}^{n} c_{k} E_{k}(x \mid \lambda)$, where

$$
c_{k}=\frac{1}{2 k !}\left\langle\left(1+\lambda e^{t}\right) t^{k} \mid p(x)\right\rangle
$$


Now, we present several applications for our theorem. As a first application, let us take $p(x)=x^{n}$ with $n \geq 0$. By Theorem 2.1, we have $x^{n}=\sum_{k=0}^{n} c_{k} E_{k}(x \mid \lambda)$, where

$$
c_{k}=\frac{1}{2 k !}\left\langle\left(1+\lambda e^{t}\right) t^{k} \mid x^{n}\right\rangle=\frac{1}{2}\left(\begin{array}{l}
n \\
k
\end{array}\right)\left\langle 1+\lambda e^{t} \mid x^{n-k}\right\rangle=\frac{1}{2}\left(\begin{array}{l}
n \\
k
\end{array}\right)\left(\delta_{n-k, 0}+\lambda\right),
$$

which implies the following identity.

Corollary 2.2 For all $n \geq 0$,

$$
x^{n}=\frac{1}{2} E_{n}(x \mid \lambda)+\frac{\lambda}{2} \sum_{k=0}^{n}\left(\begin{array}{l}
n \\
k
\end{array}\right) E_{k}(x \mid \lambda) .
$$

Let $p(x)=B_{n}(x) \in \Pi_{n}$, then by Theorem 2.1 we have that $B_{n}(x)=\sum_{k=0}^{n} c_{k} E_{k}(x \mid \lambda)$, where

$$
\begin{aligned}
c_{k} & =\frac{1}{2 k !}\left\langle\left(1+\lambda e^{t}\right) t^{k} \mid B_{n}(x)\right\rangle=\frac{1}{2}\left(\begin{array}{l}
n \\
k
\end{array}\right)\left\langle 1+\lambda e^{t} \mid B_{n-k}(x)\right\rangle \\
& =\frac{1}{2}\left(\begin{array}{l}
n \\
k
\end{array}\right)\left(B_{n-k}+\lambda B_{n-k}(1)\right),
\end{aligned}
$$

which, by (1.15), implies the following identity.

Corollary 2.3 For all $n \geq 2$,

$$
B_{n}(x)=\frac{(\lambda-1) n}{4} E_{n-1}(x \mid \lambda)+\frac{1+\lambda}{2} \sum_{k=0, k \neq n-1}^{n}\left(\begin{array}{l}
n \\
k
\end{array}\right) B_{n-k} E_{k}(x \mid \lambda) .
$$

Let $p(x)=E_{n}(x)$, then by Theorem 2.1 we have that $E_{n}(x)=\sum_{k=0}^{n} c_{k} E_{k}(x \mid \lambda)$, where

$$
\begin{aligned}
c_{k} & =\frac{1}{2 k !}\left\langle\left(1+\lambda e^{t}\right) t^{k} \mid E_{n}(x)\right\rangle=\frac{1}{2}\left(\begin{array}{l}
n \\
k
\end{array}\right)\left\langle 1+\lambda e^{t} \mid E_{n-k}(x)\right\rangle \\
& =\frac{1}{2}\left(\begin{array}{l}
n \\
k
\end{array}\right)\left(E_{n-k}+\lambda E_{n-k}(1)\right),
\end{aligned}
$$

which, by (1.18), implies the following identity.

Corollary 2.4 For all $n \geq 0$,

$$
E_{n}(x)=\frac{1+\lambda}{2} \sum_{k=0}^{n}\left(\begin{array}{l}
n \\
k
\end{array}\right) E_{n-k} E_{k}(x \mid \lambda) .
$$

For another application, let $p(x)=F_{n}(x \mid-\lambda)$, then by Theorem 2.1 we have that $F_{n}(x \mid-\lambda)=$ $\sum_{k=0}^{n} c_{k} E_{k}(x \mid \lambda)$, where

$$
\begin{aligned}
c_{k} & =\frac{1}{2 k !}\left\langle\left(1+\lambda e^{t}\right) t^{k} \mid F_{n}(x \mid-\lambda)\right\rangle=\frac{1}{2}\left(\begin{array}{l}
n \\
k
\end{array}\right)\left\langle 1+\lambda e^{t} \mid F_{n-k}(x \mid-\lambda)\right\rangle \\
& =\frac{1}{2}\left(\begin{array}{l}
n \\
k
\end{array}\right)\left(F_{n-k}(-\lambda)+\lambda F_{n-k}(1 \mid-\lambda)\right),
\end{aligned}
$$

which, by (1.21), implies the following identity. 
Corollary 2.5 For all $n \geq 1$,

$$
F_{n}(x \mid-\lambda)=\frac{1+\lambda}{2} E_{n}(x \mid \lambda)+\frac{1-\lambda^{2}}{2} \sum_{k=0}^{n-1}\left(\begin{array}{l}
n \\
k
\end{array}\right) F_{n-k}(-\lambda) E_{k}(x \mid \lambda) .
$$

Again, let $p(x)=y_{n}(x)=\sum_{k=0}^{n} \frac{(n+k) !}{(n-k) ! k !} \frac{x^{k}}{2^{k}}$ be the $n$th Bessel polynomial (which is the solution of the following differential equation $x^{2} f^{\prime \prime}(x)+2(x+1) f^{\prime}+n(n+1) f=0$, where $f^{\prime}(x)$ denotes the derivative of $f(x)$, see $\left.[3,4]\right)$. Then, by Theorem 2.1, we can write $y_{n}(x)=\sum_{k=0}^{n} c_{k} E_{k}(x \mid \lambda)$, where

$$
\begin{aligned}
c_{k} & =\frac{1}{2 k !} \sum_{\ell=0}^{n} \frac{(n+\ell) !}{(n-\ell) ! \ell ! 2^{\ell}}\left\langle 1+\lambda e^{t} \mid t^{k} x^{\ell}\right\rangle \\
& =\frac{1}{2} \sum_{\ell=k}^{n} \frac{(n+\ell) !}{(n-\ell) ! \ell ! 2^{\ell}}\left(\begin{array}{l}
\ell \\
k
\end{array}\right)\left\langle 1+\lambda e^{t} \mid x^{\ell-k}\right\rangle \\
& =\frac{1}{2} \sum_{\ell=k}^{n} \frac{(n+\ell) !}{(n-\ell) ! \ell ! 2^{\ell}}\left(\begin{array}{l}
\ell \\
k
\end{array}\right)\left(\delta_{n-k, 0}+\lambda\right) \\
& =\frac{k !}{2^{k+1}}\left(\begin{array}{c}
n \\
k
\end{array}\right)\left(\begin{array}{c}
n+k \\
k
\end{array}\right)+\lambda \sum_{\ell=k}^{n} \frac{k !}{2^{\ell+1}}\left(\begin{array}{l}
\ell \\
k
\end{array}\right)\left(\begin{array}{c}
n \\
\ell
\end{array}\right)\left(\begin{array}{c}
n+\ell \\
\ell
\end{array}\right),
\end{aligned}
$$

which implies the following identity.

Corollary 2.6 For all $n \geq 1$,

$$
y_{n}(x)=\sum_{k=0}^{n} \frac{k !}{2^{k+1}}\left(\begin{array}{l}
n \\
k
\end{array}\right)\left(\begin{array}{c}
n+k \\
k
\end{array}\right) E_{k}(x \mid \lambda)+\lambda \sum_{k=0}^{n} \sum_{\ell=k}^{n} \frac{k !}{2^{\ell+1}}\left(\begin{array}{l}
\ell \\
k
\end{array}\right)\left(\begin{array}{c}
n \\
\ell
\end{array}\right)\left(\begin{array}{c}
n+\ell \\
\ell
\end{array}\right) E_{k}(x \mid \lambda) .
$$

We end by noting that if we substitute $\lambda=0$ in any of our corollaries, then we get the well-known value of the polynomial $p(x)$. For instance, by setting $\lambda=0$, the last corollary gives that $y_{n}(x)=\sum_{k=0}^{n} \frac{(n+k) !}{(n-k) ! k !} \frac{x^{k}}{2^{k}}$, as expected.

\section{Competing interests}

The authors declare that they have no competing interests.

Authors' contributions

All authors contributed equally to the manuscript and typed, read, and approved the final manuscript.

\section{Author details}

${ }^{1}$ Department of Mathematics, Kwangwoon University, Seoul, S. Korea. ${ }^{2}$ Department of Mathematics, University of Haifa, Haifa, 3498838, Israel. ${ }^{3}$ Department of Mathematics Education, Kyungpook National University, Taegu, S. Korea. ${ }^{4}$ Division of General Education, Kwangwoon University, Seoul, S. Korea.

\section{Acknowledgements}

This paper is supported in part by the Research Grant of Kwangwoon University in 2013

Received: 3 April 2013 Accepted: 10 October 2013 Published: 08 Nov 2013

\section{References}

1. Kim, DS, Kim, T: Applications of umbral calculus associated with $p$-adic invariant integrals on $\mathbb{Z}_{p}$. Abstr. Appl. Anal. 2012, Article ID 865721 (2012)

2. Kim, DS, Kim, T: Some identities of Frobenius-Euler polynomials arising from umbral calculus. Adv. Differ. Equ. 2012, Article ID 196 (2012) 
3. Roman, S: More on the umbral calculus, with emphasis on the $q$-umbral calculus. J. Math. Anal. Appl. 107, 222-254 (1985)

4. Roman, S: The Umbral Calculus. Dover, New York (2005)

5. Kim, T: Identities involving Frobenius-Euler polynomials arising from non-linear differential equations. J. Number Theory 132(12), 2854-2865 (2012)

6. Robinson, TI: Formal calculus and umbral calculus. Electron. J. Comb. 17(1), \#R95 (2010)

7. Bayad, A, Kim, T: Results on values of Barnes polynomials. Rocky Mt. J. Math. Forthcoming Articles (2013)

8. Kim, T: Symmetry p-adic invariant integral on $\mathbb{Z}_{p}$ for Bernoulli and Euler polynomials. J. Differ. Equ. Appl. 14(279), 1267-1277 (2008)

9. Tremblay, R, Gaboury, S, Fugére, B-J: Some new classes of generalized Apostol-Euler and Apostol-Genocchi polynomials. Int. J. Math. Math. Sci. 2012, Article ID 182785 (2012)

10. Kim, T: Some identities on the $q$-Euler polynomials of higher order and $q$-Stirling numbers by the fermionic $p$-adic integral on $\mathbb{Z}_{p}$. Russ. J. Math. Phys. 16, 484-491 (2009)

11. Araci, S, Acikgoz, M: A note on the Frobenius-Euler numbers and polynomials associated with Bernstein polynomials. Adv. Stud. Contemp. Math. 22(3), 399-406 (2012)

12. Bayad, A, Kim, T: Identities involving values of Bernstein, $q$-Bernoulli, and $q$-Euler polynomials. Russ. J. Math. Phys. 18(2), 133-143 (2011)

13. Kim, T: An identity of the symmetry for the Frobenius-Euler polynomials associated with the fermionic $p$-adic invariant $q$-integrals on $\mathbb{Z}_{p}$. Rocky Mt. J. Math. 41, 239-247 (2011)

14. Ding, D, Yang, J: Some identities related to the Apostol-Euler and Apostol-Bernoulli polynomials. Adv. Stud. Contemp. Math. 20(1), 7-21 (2010)

15. Bayad, A, Kim, T: Identities for the Bernoulli, the Euler and the Genocchi numbers and polynomials. Adv. Stud. Contemp. Math. 20(2), 247-253 (2010)

16. Carlitz, L: The product of two Eulerian polynomials. Math. Mag. 23, 247-260 (1959)

17. Carlitz, L: The product of two Eulerian polynomials. Math. Mag. 36, 37-41 (1963)

18. Can, M, Cenkci, M, Kurt, V, Simsek, Y: Twisted Dedekind type sums associated with Barne's type multiple Frobenius-Euler L-functions. Adv. Stud. Contemp. Math. 18(2), 135-160 (2009)

19. Cangul, IN, Kurt, V, Ozden, H, Simsek, Y: On the higher-order w-q-Genocchi numbers. Adv. Stud. Contemp. Math. 19(1), 39-57 (2009)

10.1186/1687-1847-2013-301

Cite this article as: Kim et al.: Apostol-Euler polynomials arising from umbral calculus. Advances in Difference Equations 2013, 2013:301

\section{Submit your manuscript to a SpringerOpen ${ }^{\ominus}$ journal and benefit from:}

- Convenient online submission

- Rigorous peer review

- Immediate publication on acceptance

- Open access: articles freely available online

- High visibility within the field

- Retaining the copyright to your article 